HIAS-E-21

\title{
Is Diversity in Capabilities Desirable When Adding Decision Makers?
}

\author{
Ruth Ben-Yashar \\ Department of Economics, Bar Ilan University, Ramat Gan, Israel \\ Shmuel Nitzan \\ Department of Economics, Bar Ilan University, Ramat Gan, Israel \\ Hitotsubashi Institute for Advanced Study, Hitotsubashi University, Japan
}

March 16, 2016

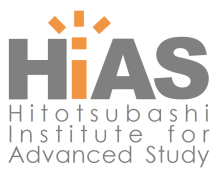

Hitotsubashi Institute for Advanced Study, Hitotsubashi University 2-1, Naka, Kunitachi, Tokyo 186-8601, Japan

tel:+81 425808604 http://hias.ad.hit-u.ac.jp/

HIAS discussion papers can be downloaded without charge from: http://hdl.handle.net/10086/27202

https://ideas.repec.org/s/hit/hiasdp.html

All rights reserved. 
Is diversity in capabilities desirable when adding decision makers? ${ }^{+}$

by

Ruth Ben-Yashar** and Shmuel Nitzan*

\begin{abstract}
When the benefit of making a correct decision is sufficiently high, even a slight increase in the probability of making such a decision justifies an increase in the number of decision makers. Applying a standard uncertain dichotomous choice benchmark setting, this study focuses on the relative desirability of two alternatives: adding individuals with capabilities identical to the existing ones and adding identical individuals with mean-preserving capabilities that depend on the states of nature. Our main result establishes that when the group applies the simple majority rule, variability in the capabilities of the new decision makers under the two states of nature, which is commonly observed in various decision-making settings, is less desirable in terms of the probability of making the correct decision.
\end{abstract}

Keywords: decisional capabilities, group extension, asymmetry, homogeneity, diversity, mean preservation

JEL Classification number: D81

*Department of Economics, Bar Ilan University, Ramat Gan , Israel and Hitotsubashi Institute of Advanced Studies, Hitotsubashi University.

**Department of Economics, Bar Ilan University, Ramat Gan, Israel

+The authors are indebted to two anonymous referees and an associate editor for their most useful comments and suggestions. 


\section{Introduction}

A sufficiently high benefit of making a correct decision may justify an increase in the number of decision makers that increases, even slightly, the probability of making such a decision. Applying a standard uncertain dichotomous choice benchmark setting, with identical independent symmetric decisional capabilities that are invariant to the state of nature, our objective is to compare the relative desirability of two alternatives: adding individuals with symmetric capabilities identical to the existing ones and adding identical individuals with asymmetric mean-preserving capabilities that differ in the two states of nature. Under the first alternative, the independent decisional capabilities of the group members remain homogeneous and invariant to the state of nature. Under the second strategy, the decisional skills of the new group members are identical, but depend on the state of nature. ${ }^{1}$

Along time decision makers tend to become equally skilled due to the accumulated common experience and the exposure to effective learning processes. Skill uniformity can be changed, at least temporarily, by injecting "new blood" to the group, namely by adding new members. The added members can be of different skills than the existing members, or even be identical to each other, but depend on the state of nature. In the latter case, the probability of a correct decision is higher in one state of nature than in the other.

According to Condorcet Jury Theorem (CJT), Condorcet (1785), if the identical symmetric decision makers vote independently and each of them has the same probability $p>1 / 2$ of making the correct choice, then the likelihood of a correct decision is increasing with the number of decision makers. ${ }^{2}$ This means that the first

\footnotetext{
${ }^{1}$ Sah (1991), Sah and Stiglitz (1988) relaxed the symmetry assumption with respect to the states of nature and allowed the decisional skills of each voter to depend on the state of nature. Ben-Yashar and Nitzan (1997) derived the optimal group decision rule under such asymmetric setting. Ben-Yashar (2014) reassesses the validity of the Condorcet Jury Theorem when voters are homogeneous and each knows the correct decision with an average probability of more than a half. This paper shows that larger groups, in most cases, are less likely to reach a correct collective decision, even if the average individual probability of making a correct decision exceeds one half.

${ }^{2}$ CJT has previously been generalized in several other ways. Early expositions and generalizations were proposed by Grofman et al. (1983), Feld and Grofman (1984), Nitzan and Paroush (1982), Young (1988) and Owen et al. (1989). Ladha (1995) relaxed the independence assumption. Austen-Smith and Banks (1996) and Ben-Yashar and Milchtaich (2007) generalized the setting to a strategic one. CJT can be generalized to the case of heterogeneous voters. See, for example, Ben-Yashar and Zehavi (2011), Ben-Yashar and Danziger (2011) and Berend and Paroush (1998). Baharad and Ben-Yashar (2009) studied the validity of CJT under subjective probabilities. Dietrich and List (2013) presented a general
} 
alternative is advantageous when the decision makers remain homogeneous and invariant to the state of nature, but may differ in their judgments regarding the identity of that choice. In many decision-making contexts, the decisional capabilities corresponding to the two possible states of nature often differ. Under the second possible alternative that we consider, each of the two identical added members is associated with two probabilities of voting correctly that correspond to the two possible states of nature, such that their average is equal to the single probability of making a correct decision that represents the ability of each of the existing group members. The question is whether such mean-preserving variability in the capabilities of the added identical decision makers is more or less desirable than the first alternative. It turns out that the first alternative is superior to the second one.

Note that another possible alternative is to add individuals with different mean-preserving capabilities. In such a case, the decisional skills of the new group members are different, yet still independent of the state of nature. Diversifying capabilities in this sense is the best possibility in terms of its effect on the likelihood of making a correct choice. Ben- Yashar and Paroush (2000) show that this meanpreserving alternative outperforms the addition of homogeneous decision makers, the skills of whom are identical to that of the group members. This paper's main result, according to which the addition of homogenous members outperforms the addition of members whose skills are identical to the ones of the group members yet depend on the state of nature, combined with Ben-Yashar and Paroush (2000) result, implies that the best alternative is adding heterogeneous members.

A possible application of our model is a panel of homogeneous (skill-wise) judges. The judges are classified as "seniors" due to their expertise and experience. When adding unexperienced judges, who are referred to as "juniors", they may obtain different specializations such that different skills are associated with different states of nature. Another application is a situation where the correct decision is obtained after examining a number of tests or criteria. Every such criterion may identify the correct alternative with a given probability. One may perceive this probability as the "criterion's skill". When it is possible to add some criteria, our result suggests that one should add two new symmetric criteria of equal quality to the existing ones rather

analysis of proposition-wise judgment aggregation. For a recent survey of the literature inspired by CJT see Nitzan and Paroush (2015). 
than adding two new mean-preserving, identical, asymmetric criteria that are superior in one state and inferior in the other state relative to the existing ones. For example, in critical medical, environmental and earthquake or tsunami preventive diagnostic decisions, classic distinction is symmetric, namely, independent of the state of nature. This is the case with cancer tests based on biopsy and with non-invasive procedures such as ultrasound, MRI and CT. The reason that these diagnostic means are symmetric is that they do not focus on a particular attribute of the disease but take into account all or most of the attributes of the disease. The new studies are concerned with the detection of a disease by taking into account one of its attributes' -- tests that focus on early detection, less invasive tests and on lower cost tests. In this case asymmetric capabilities are commonly observed so the probability of a correct decision is higher in one state of nature than in the other. This is the case with genebased diagnostic systems for early detection of certain types of cancer. The same is true for the PSA test for the early detection of prostate cancer. These systems are typically more reliable in diagnosing a patient who is sick than in making a correct diagnosis when the patient is healthy. The reason is that cancer has several possible origins and the common diagnostic means mentioned above focus on one of these, so the probability of a correct decision is higher in one state of nature (the patient having cancer) than in the other (the patient not having cancer).

\section{The model}

Let the decision-making group consists of $n=2 k+1$ members, $k$ being a positive integer. $^{3}$ Each individual selects one of two alternatives. The two possible equiprobable states of nature are denoted by 1 and 2 . A voter chooses the correct alternative with probability $p_{1}\left(p_{2}\right)$ in state of nature $1(2)$. We assume that $p_{1}>1$ $p_{2}$; that is, a voter is more likely to decide 1 in state 1 than in state 2 , which implies that the average of the voter's probabilities in the two states of nature exceeds $1 / 2$. Note that all individuals share the same objective of selecting the correct alternative. ${ }^{4}$ Individual probabilities of voting for the correct alternative are not statistically

\footnotetext{
${ }^{3}$ Since we consider a choice between two alternatives using the simple majority rule, we require an odd number of decision makers. The rule is not defined otherwise.

${ }^{4}$ Within our framework the objective is to make the right decision, which is equivalent to maximizing the expected payoff when symmetric payoffs are assumed.
} 
correlated and the simple majority rule is used to aggregate votes to determine the collective decision.

According to the common version of CJT, group members are assumed to be homogeneous and symmetric, i.e., possess identical decisional skills that are equal in the two states of nature, $p$, such that $1 / 2<p=p_{1}=p_{2}$. Let $\pi(p, n)$ denote the probability of the group to decide correctly under the simple majority rule. Formally: $\pi(p, n)=\sum_{i=k+1}^{2 k+1}\left(\begin{array}{c}2 k+1 \\ i\end{array}\right) p^{i}(1-p)^{2 k+1-i}$

The probability of reaching a correct decision under simple majority rule is equal to the sum of the probabilities of the majority making a correct bdecision. That is, when at least $k+1$ members support the same correct alternative.

By isolating two members from the original group, following Ben-yashar and Paroush (2000), we can rewrite $\pi(p, n)$ as: $\pi(p, n)=p^{2} A+\left(1-(1-p)^{2}\right) B+C$, where

$$
A=\left(\begin{array}{c}
2 k-1 \\
k-1
\end{array}\right) p^{k-1}(1-p)^{k}, B=\left(\begin{array}{c}
2 k-1 \\
k
\end{array}\right) p^{k}(1-p)^{k-1} \text { and } C=\sum_{i=k+1}^{2 k-1}\left(\begin{array}{c}
2 k-1 \\
i
\end{array}\right) p^{i}(1-p)^{2 k-1-i}
$$

Suppose now that the above two members decide correctly. Hence, in order to obtain a majority, $k-1$ out of $2 k-1$ are required to decide correctly. This requirement is described in term A. When at least one of the two members decide correctly, then a majority is obtained if $k$ out of $2 k-1$ decide correctly, which is reflected in B. Finally, term $\mathrm{C}$ presents the sum of probabilities for obtaining a majority of at least $k+1$ out of the $N-1$ members. The probability that the group chooses the correct alternative without the two members (i.e., a group of $2 k-1$ members) is $\mathrm{B}+\mathrm{C}$.

Therefore, adding two homogeneous members improves the performance of the group iff

$p^{2} A+\left(1-(1-p)^{2}\right) B+C>B+C \Leftrightarrow p^{2} A-(1-p)^{2} B>0$.

This condition is always satisfied since $\frac{B}{A}=\frac{p}{1-p}$ and, indeed, by CJT, under symmetric homogeneous valuable independent decisional skills the marginal extension of a group is always advantageous. 
When each of the two identical added members is associated with two probabilities $p_{1}$ and $p_{2}$ of voting correctly under the two possible states of nature 1 and 2, respectively, the performance of the existing homogeneous group is improved iff

$0.5\left(p_{1}^{2} A+\left(1-\left(1-p_{1}\right)^{2}\right) B+C+p_{2}^{2} A+\left(1-\left(1-p_{2}\right)^{2}\right) B+C\right)>B+C \Leftrightarrow$ $\left(\left(p_{1}^{2}+p_{2}^{2}\right) A-\left(\left(1-p_{1}\right)^{2}+\left(1-p_{2}\right)^{2}\right) B\right) 0.5>0 \Leftrightarrow$

$\frac{p}{1-p}<\frac{p_{1}^{2}+p_{2}^{2}}{\left(1-p_{1}\right)^{2}+\left(1-p_{2}\right)^{2}}$.

\section{Result}

The increase in the collective probability of making the correct choice due to the addition of two identical members that are equally skilled to the existing members is denoted by $\Delta_{h s}$ (the index hs refers to homogeneous skills). By (1), $\Delta_{h s}$ is given by: $\Delta_{h s}=p^{2} A-(1-p)^{2} B$.

The increase in the collective probability of making the correct choice due to the expansion of the existing homogeneous group by two identical members with asymmetric skills that depend on the state of nature is denoted by $\Delta_{\text {has }}$ (the index has refers to homogeneous asymmetric state-dependent skills). By (2), $\Delta_{\text {has }}$ is given by:

$$
\Delta_{\text {has }}=0.5 A\left(p_{1}^{2}+p_{2}^{2}\right)-0.5 B\left(\left(1-p_{1}\right)^{2}+\left(1-p_{2}\right)^{2}\right) \text {. }
$$

To compare the marginal effects of group extension we have considered above, suppose that the addition of asymmetric members is mean-preserving. That is, the average decisional skills of the added identical asymmetric members is equal to the decisional skill of the existing homogeneous symmetric members, $p=\frac{p_{1}+p_{2}}{2}$.

Let $\Delta_{h t s}=p_{1} p_{2} A-\left(1-p_{1}\right)\left(1-p_{2}\right) B . \quad \Delta_{h t s}$ represents the increase in the collective probability of making the correct choice due to the extension of the existing homogeneous group by two different members, one with probability $p_{1}$ in the two 
states of nature and the other with probability $p_{2}$ in the two states of nature (here the index hts refers to heterogeneous skills).. ${ }^{5}$

Formally

$p_{1} p_{2} A+\left(1-\left(1-p_{1}\right)\left(1-p_{2}\right)\right) B+C-(B+C) \equiv p_{1} p_{2} A-\left(1-p_{1}\right)\left(1-p_{2}\right) B$

This expression is always positive (Ben-Yashar and Paroush (2000)) provided that the average skill of the added members is at least equal to the homogeneous skill of the existing group members.

Suppose that the addition of members is mean-preserving. That is, the average decisional skills of the added different members or of the added identical asymmetric members are equal to the ones of the existing homogeneous symmetric members, that is, $p=\frac{p_{1}+p_{2}}{2}$.

\section{Theorem 1:}

If $p=\frac{p_{1}+p_{2}}{2}$, then $\Delta_{h t s}>\Delta_{h s}>\Delta_{h a s}$.

Proof:

$$
\begin{aligned}
& \Delta_{h s}>\Delta_{h a s} \Leftrightarrow \\
& p^{2} A-(1-p)^{2} B>0.5 A\left(p_{1}^{2}+p_{2}^{2}\right)-0.5 B\left(\left(1-p_{1}\right)^{2}+\left(1-p_{2}\right)^{2}\right) \Leftrightarrow \\
& A\left(p^{2}-\frac{p_{1}^{2}+p_{2}^{2}}{2}\right)-B\left((1-p)^{2}-\frac{\left(1-p_{1}\right)^{2}+\left(1-p_{2}\right)^{2}}{2}\right)>0 \Leftrightarrow \\
& A\left(p^{2}-\frac{p_{1}^{2}+p_{2}^{2}+2 p_{1} p_{2}}{2}+p_{1} p_{2}\right)- \\
& B\left((1-p)^{2}-\frac{\left(1-p_{1}\right)^{2}+\left(1-p_{2}\right)^{2}+2\left(1-p_{1}\right)\left(1-p_{2}\right)}{2}+\left(1-p_{1}\right)\left(1-p_{2}\right)\right)>0 \Leftrightarrow
\end{aligned}
$$

\footnotetext{
${ }^{5}$ Note that in this case the probabilities $p_{1}$ and $p_{2}$ are of different individuals, whereas in (2) the different probabilities $p_{1}$ and $p_{2}$ represent the skills of a single individual under two states of nature.
} 


$$
\begin{aligned}
& A\left(p^{2}-\frac{\left(p_{1}+p_{2}\right)^{2}}{2}+p_{1} p_{2}\right)- \\
& B\left((1-p)^{2}-\frac{\left(2-\left(p_{1}+p_{2}\right)\right)^{2}}{2}+\left(1-p_{1}\right)\left(1-p_{2}\right)\right)>0 \Leftrightarrow
\end{aligned}
$$

$$
\begin{aligned}
& A\left(p^{2}-\frac{4 p^{2}}{2}+p_{1} p_{2}\right)- \\
& B\left((1-p)^{2}-\frac{(2-2 p)^{2}}{\left(-p^{2}+p_{1} p_{2}\right)-2 B\left(-(1-p)^{2}+\left(1-p_{1}\right)\left(1-p_{2}\right)\right)>0 \Leftrightarrow}\right)>0 \Leftrightarrow
\end{aligned}
$$$$
p_{1} p_{2} A-B\left(1-p_{1}\right)\left(1-p_{2}\right)>-B(1-p)^{2}+A p^{2} \text {. }
$$

By Ben-yashar and Paroush (2000) $\Delta_{h t s}>\Delta_{h s}$ which implies that

$p_{1} p_{2} A-\left(1-p_{1}\right)\left(1-p_{2}\right) B>p^{2} A-(1-p)^{2} B$, which is the same as (4).

This completes the proof.

\section{Discussion}

The result of Ben-Yashar and Paroush (2000), namely the superiority of marginal group extension by different individuals with symmetric mean-preserving decisional skills, $\Delta_{h t s}>\Delta_{h s}$, is due to the so called "dimensionally strict monotonicity" of the function specifying the collective probability of making a correct choice using the simple majority rule. By this property, the effect of the improved individual skill is stronger than the effect of the reduced individual skill. On the other hand, our result, $\Delta_{h s}>\Delta_{h a s}$ namely the relative inferiority of marginal group extension by identical individuals with asymmetric mean-preserving decisional skills that depend on the state of nature, is due to the fact that the marginal change in the collective probability of making a correct choice using the simple majority rule is declining with respect to the decisional quality of the two added members. Hence, the positive effect of the 
improved individual skills in one state of nature is more than counter balanced by the negative effect of the reduced individual skills in the other state of nature. ${ }^{6}$

The relevance of the assumption of symmetric prior (i.e. symmetric alternatives) is due to the unbiasedness required from the final decisions, which is essential in courts decisions. Clearly, if the environment is not perfectly symmetric, it is possible to obtain a different result. For instance, suppose that the a- priori probability of one state is relatively high. In such a case, the addition of decision makers who are highly skilled in this state, would clearly be advantageous. In this case, the inequality is reversed, i.e.. $\Delta_{h s}<\Delta_{h a s}{ }^{7}$

Our results are not robust to the addition of members with different skills. Suppose that the two states of nature are characterized by similar parameters, then it is optimal that the two members balance each other, in the sense that one is more skilled in one state and the other in the other state. As a special case, consider adding two different individuals whose skills depend on the state of nature, one with probability of $p+d$ in state 1 and of $p$-d in state 2 , and the other member with probability $p$ - $d$ in state 1 and $p+d$ in state 2 , which is still mean-preserving. This case is equivalent (probability wise) to adding two heterogeneous individuals; yet, as we have shown, it is advantageous. That is,

$$
\begin{aligned}
& 0.5((p+d)(p-d) A+(1-(1-p-d)(1-p+d)) B+C+(p-d)(p+d) A+(1-(1-p+d)(1-p-d)) B+ \\
& (p+d)(p-d) A+(1-(1-p-d)(1-p+d)) B .
\end{aligned}
$$

which is equal to $\Delta_{h t s}$.

\section{References}

\footnotetext{
${ }^{6}$ Notice that this economic intuition is not valid for explaining the former result of Ben-Yashar and Paroush (2000). The reason is that in their case, in each state of nature the added decisional capabilities of the two individuals are different. Since the positive marginal effect requires the use of both of these different capabilities, it is meaningless to resort to the notion of declining marginal productivity.

${ }^{7}$ To analyze, let $a$ be the apriory probability of state 1 . In this case

$\Delta_{\text {has }}=A\left(a p_{1}^{2}+(1-a) p_{2}^{2}\right)-B\left(a\left(1-p_{1}\right)^{2}+(1-a)\left(1-p_{2}\right)^{2}\right)$

If $a$ is close to 1 and $p_{1}$ is higher than $p_{2}$, then $\Delta_{h s}<\Delta_{h a s}$. Alternatively, one can add a parameter $q$ such that $p_{1}=p+q$ and $p_{2}=p-q$, and look for the optimal value of $q$. When the prior is completely symmetrical one can obtain $q=0$,i.e, symmetric skills. As far as a priori probability stay away from half than the optimum value of $q$ is considerably greater than 0 .
} 
Austen-Smith, D., and Banks, J. (1996): "Information Aggregation, Rationality, and the Condorcet Jury Theorem", American Political Science Review 90: 34-45.

Baharad, E. and Ben-Yashar, R. (2009), "The Robustness of the Optimal Weighted Majority Rule to Probabilities Distortion”, Public Choice 139, 53-59.

Ben-Yashar, R. (2014): "The Generalized Homogeneity Assumption and the Condorcet Jury Theorem", Theory and Decision, 77, 237-241.

Ben-Yashar, R. and Danziger , L. (2011): "Symmetric and asymmetric committees", Journal of Mathematical Economics 47, 440-447.

Ben-Yashar, R., and Milchtaich, I. (2007): "First and Second Best Voting Rules in Committees", Social Choice and Welfare 29(3): 453-480.

Ben-Yashar, R., and Nitzan, S. (1997): “The Optimal Decision Rule for Fixed-Size Committees in Dichotomous Choice Situations: The General Result", International Economic Review 38(1): 175-186.

Ben-Yashar, R. and Paroush, J. (2000), “A Nonasymptotic Condorcet Jury Theorem”, Social Choice and Welfare 17, 189-199.

Ben-Yashar, R., and Zahavi, M., (2011), "The Condorcet Jury Theorem and Extension of the Franchise with Rationally Ignorant Voters", Public Choice $148,435-443$.

Berend, D., \& Paroush, J. (1998), When is Condorcet's Jury Theorem valid? Social Choice and Welfare, 15, 481-488.

Condorcet, N.C. de. (1785), Essai sur l'Application de l'Analyse à la Probabilité des Décisions Rendues à la Pluralité des Voix, Paris. See I. McLean and F. Hewitt, translators, 1994.

Dietrich, F. and List, C. (2013), "Propositionwise Judgment Aggregation: The General Case", Social Choice and Welfare 40, 1067-1095.

Feld, S.L. and Grofman, B. (1984), “The Accuracy of Group Majority Decisions in Groups with Added Members”, Public Choice 42, 273-285. 
Grofman, B., Owen, G. and Feld, S.L. (1983): "Thirteen theorems in search of truth", Theory and Decision 15, 261-278.

Ladha, K.K. (1995): "Information Pooling through Majority-Rule Voting: Condorcet's Jury Theorem with Correlated Votes", Journal of Economic Behavior and Organization 26: 353-372.

Nitzan, S. and Paroush, J. (1982), "Optimal Decision Rules in Uncertain Dichotomous Choice Situation", International Economic Review 23, 289-297.

Nitzan, S. and Paroush, J. (2015), Collective Decision Making and Jury Theorems, Oxford Handbook of Law and Economics. Oxford University Press, forthcoming.

Owen, G.B.,\& Grofman, B., Feld S.L. (1989). Proving a distribution-free generalization of the Condorcet Jury Theorem. Mathematical Social Sciences, 17: 1-16.

Sah, R.K. (1991): "Fallibility in Human Organizations and Political Systems", The Journal of Economic Perspectives 5: 67-88.

Sah, R.K. and Stiglitz, J.E. (1988): "Committees, Hierarchies and Polyarchies", The Economic Journal 98: 451-470.

Young, H.P. (1988): “Condorcet's Theory of Voting“, American Political Science Review 82: 1231-1244. 